\title{
Evaluation of Artificial Neural Networks in Prediction of Essential Hypertension
}

\author{
Rahul Samant \\ SVKM's NMIMS \\ Shirpur, MH 425405 India
}

\author{
Srikantha Rao \\ TIMSCDR \\ Kandivali, Mumbai MH 400101 India
}

\begin{abstract}
This paper investigates the ability of variously designed \& trained Artificial Neural Network (ANN) to predict the probability of occurrence of Hypertension (HT) in a mixed (healthy + hypertensive, both sexes) patient population. To do this a multi layer feed-forward neural network with 13 inputs and 1 output was created with multiple hidden layers. Network parameters such as count of hidden layers, count of neurons in the hidden layers, percentage of testing samples and percentage of samples used for validation were varied so as to deliver the maximum prediction accuracy of the ANN network. The training algorithm used for ANN is LevenbergMarquardt back propagation algorithm. A large database, comprising healthy and hypertensive patients from a university hospital was used for training the ANN and prediction. The maximum accuracy marked by this approach was $92.85 \%$, considered quite satisfactory by medical experts. Thus the best network parameter choice best for ANNs approached empirically.
\end{abstract}

\section{General Terms}

ANN Classification, Diagnosis

\section{Keywords}

Artificial Neural Network, medical diagnosis, essential hypertension, accuracy.

\section{INTRODUCTION}

In the practice of medicine, Artificial Neural Networks are now being vigorously applied in areas as diverse as cytology, cardiology, analysis of images, genetics and clinical chemistry. [1] However the major problem here is to diagnose a disease with high reliability. Human experts make mistakes because of their diverse experiences, training and other limitations. This is the major issue for medical practitioners. One of the most important problems of medical diagnosis is the subjectivity of the specialist $[13,15]$.This is due to the fact that the result does not depend on a systematized solution but on the interpretation of the patient's signals [5]. Brause highlighted that almost all physicians are confronted during their training by the difficult task of diagnosing a disease. Here, they have to solve the problem of deducing certain diseases or formulating a treatment based on more or less specified symptoms observed and medical knowledge. Principally, human thinker does not resemble statistical modeling as done with computers but it acts like pattern recognition systems. In particular, it has been repeatedly noted that Humans can recognize patterns or objects very easily but fail when probabilities have to be assigned to different outcomes, here diagnosis. In reality, the quality of diagnosis is totally depended on the physician talent and experience. Further, emotional problems and fatigue degrade the doctor's performance. The training procedure of doctors, in particular specialists, is a lengthy and expensive one. So even in developed countries one may sometimes feel the lack of sound medical advice. Medical science is one of the most rapidly growing and changing fields of science. Therefore like many other endeavors of modern enterprise, today knowledge-based technology is being increasingly engaged in the field of medical diagnosis.[4]

\section{LITERATURE REVIEW}

Ture, Kurt, Kurum, Ozdamar [8] compared performances of two neural networks, four statistical algorithms and three decision trees in order to predict the risk of essential hypertension disease. MLP and RBF - two neural networks procedures-performed better than other techniques in predicting hypertension. Gil, Johnsson, Garicia, Paya and Fernandez [7] developed two types of unsupervised and one supervised neural network to evaluate the use of neural network models as a tool to support diagnosis of urological dysfunctions. Hsu, Chiu, Lee, Chiu, Liu and Hwang [9] constructed a classification approach based on the hybrid use of case-based reasoning (CBR) and genetic algorithms (GAs). Hypertension detection was attempted using anthropometric body surface scanning data. The obtained result revealed the relationship between a subject's 3D scanning data and hypertension disease. GA was adopted to determine the optimum feature weights for CBR. The proposed approaches were compared with a regular CBR and other widely used approaches including neural nets and decision trees. Zhang, Yan, Zhao and Zhang [10] developed an ANN based automated computer aided diagnosis system to help radiologist in detecting micro-calcifications in digital format mammograms. Dana [14] developed a ANN based AI system to detect breast cancer. The system was trained using eight input nodes represent features of calcification, areas in breast tissues where tiny calcium deposits built up and might indicate the presence of cancer.

\section{ARTIFICIAL NEURAL NETWORK}

An artificial neural network (ANN) is a computational model that attempts to functionally mimic human brain [2]. It is a network of highly interconnected processing elements (neurons) operating in parallel. These elements are similar to the human nervous system. A subgroup of processing elements is called a layer in the ANN network. The first layer of a ANN is the input layer and the last layer of a ANN is the output layer. There may be additional layers called hidden layers. Fig.1 represents the typical neural network. A feed forward neural network can be trained to perform a particular function by adjusting the values of the weights between elements. Medical diagnosis using ANN is currently a very active research area in medicine and it is believed that it can be more widely used in biomedical systems in the next few years [14]. 
Input Values

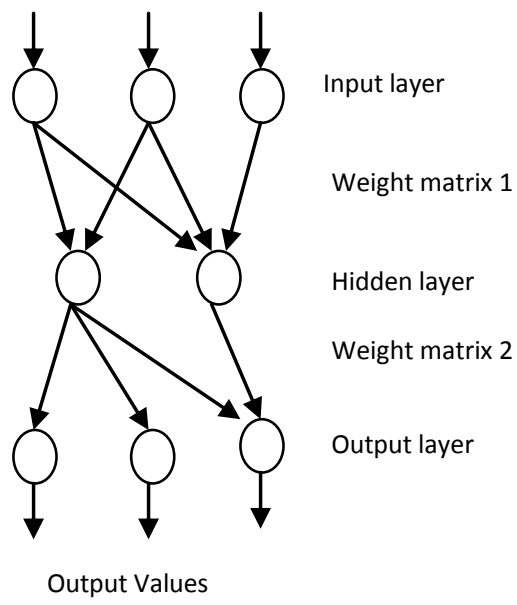

Fig 1: A typical neural network

This is primarily because the solution is not restricted to linear form. Neural networks are ideal in recognizing diseases using health parameters since there is no need to provide a specific algorithm on how to identify the disease. Neural networks learn by examples so the details of how to recognize the disease is not needed [13].

\section{ANN-BASED DIAGNOSIS MODEL}

Neural networks are widely and successfully used techniques for classification and prediction. All types of back propagation neural networks are proposed to diagnose diseases [3]. The model consists of three layers: the input layer, a hidden layer and the output layer. For the present study, a single hidden layer with 20 neurons was created and trained. The transfer function used was 'tansig'. We used 'trainlm' function for back-propagation network training and 'learngdm' function for back-propagation weight/bias learning. These were default options provided in the tool. The input and target samples were divided into validation, testing and training sets. The training set is used to train the network. The test set provides a completely independent measure of network accuracy. The information moves is forward direction from the input nodes through hidden layers to output nodes. The schematic of the neural network model built are shown in Fig. 2

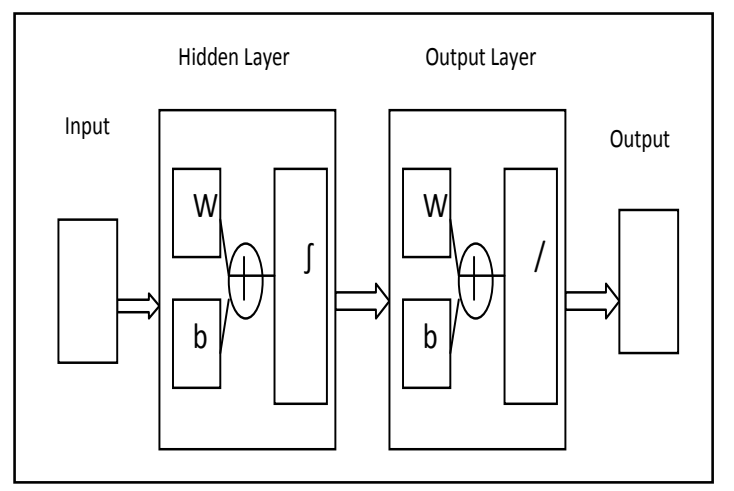

Fig 2: A typical ANN model for hypertension prediction
A feed-forward neural network allows signals to travel in only one direction, from input to output. The hidden neurons are able to learn the patterns in data during the training phase and mapping the relationship between input and output pairs. Data received from the input layer is processed by each neuron in the hidden layer using a transfer function to the output neuron for further processing.

The output of the hidden layer can be explained by equation (1)

$Y N x 1=f(W N x M X M, 1+b N, 1)$

Where $Y$ is a vector of the output from each of the $N$ neurons in a given layer, $W$ is a matrix containing the weights for each of the $M$ inputs for all $N$ neurons, $X$ is a vector of inputs, $b$ is a vector of biases and $f($.$) is the activation function. [2]$

\section{METHODOLOGIES EMPLOYED}

\subsection{Data Acquisition and Preprocessing}

The database used for analysis in this study has been compiled as a part of an earlier study entitled Early Detection Project (EDP) conducted at the Hemorheology Laboratory of the erstwhile Inter-Disciplinary Programme in Biomedical Engineering at the School (now Department) of Biosciences and Bioengineering, Indian Institute of Technology Bombay (IITB), Mumbai, India. Spanning over a period from January 1995 to April 2005, it compiled 981 records, each with 13 parameters, which encapsulated the biochemical, hemorheological and clinical status of the individuals- mainly students, faculty, staff and their family members visiting IITB Hospital for routine checkups or treatment of common ailments. We note that the Hemorheology Laboratory has pioneered the research in the field of Clinical Hemorheology by conducting the baseline hemorheological studies in the Indian population and correlating various hemorheological parameters with several disease conditions.

The diagnosis of hypertension is made by a doctor as follows. When the average of three or more diastolic blood pressure (BP) measurements on at least three subsequent visits is R90 $\mathrm{mmHg}$ or when the average of multiple systolic BP readings on three or more subsequent visits is consistently R140 $\mathrm{mmHg}$ [9], the patient is diagnosed to have hypertension.

In all, 13 parameters were noted for each respondent [17]. Table 1 describes the symptom (input) variables used for the present study. They include age, health indicators (e.g. pulse, systolic blood pressure (BP1), diastolic blood pressure (BP2)) and biochemical parameter like Serum Proteins (SP), Serum Albumin (SALB), Hematocrit (HCT), Erythrocyte Sedimentation Rate (ESR), Serum Cholesterol (SC), Serum Triglycerides (STG), along with various hemorheological (HR) parameters (e.g.; Whole Blood Viscosity-WBVmeasured over eight different shear rates, Plasma Viscosity$\mathrm{PV}$-measured over three different shear rates, using a Contraves 30 viscometer, and Red Cell Aggregation (RCA).

We studied the effect of four network parameters on the prediction accuracy of an ANN. Table 1 lists the effect of varying neuron count in the hidden layer of two-layer ANN. Dataset used in our study is KNN-imputed dataset for missing values at random, where missing data percentage is less than $15 \%$ [16]. The dataset consists of information about patients who are hypertensive and healthy as diagnosed by the doctors. 
Table 1. Diagnosis variables of datasets used in the study

\begin{tabular}{|c|c|c|}
\hline Num. & $\begin{array}{c}\text { Symptom } \\
\text { variable name }\end{array}$ & Data Type \\
\hline 1. & AGE & Numeric, Range(19-73) \\
\hline 2. & BSF & Numeric, Range $(48,311)$ \\
\hline 3. & BSP & Numeric, Range $(61,383)$ \\
\hline 4. & SC & Numeric, Range(90,389) \\
\hline 5. & STG & Numeric, Range $(41,456)$ \\
\hline 6. & SALB & Numeric, Range $(3.1,6.45)$ \\
\hline 7. & SP & Numeric, Range $(0.83,10.68)$ \\
\hline 8. & CPV2 & Numeric, Range $(1.069,1.785)$ \\
\hline 9. & CB2 & Numeric, Range $(2.448,8.695)$ \\
\hline 10. & HCT & Numeric, Range(22,60) \\
\hline 11. & RG & Numeric, Range $(1.374,6.174)$ \\
\hline 12. & BP1 & Numeric, Range(98,240) \\
\hline 13. & BP2 & Numeric, Range(60,116) \\
\hline
\end{tabular}

\section{RESULTS AND DISCUSSION}

The neural network toolbox from Matlab R2007a was used to build a variety of diagnosis models for predicting hypertension from symptom data and evaluate their classification accuracy. In the beginning a single hidden layer with 20 neurons was created and trained with $80 \%$ training samples, $10 \%$ testing samples and $10 \%$ validating samples randomly taken from the dataset. The well established Levenberg-Marquardt back propagation algorithm was used to train the network. The results of applying the ANN to classify between healthy and unhealthy patients based on selected symptoms showed very good ability of the network to learn the patterns (relationships to sickness) corresponding to the symptoms of the person. The best network configuration appears to be having 20 neurons in first hidden layer and 5 neurons in the second hidden layer. A small sample set (10\%) for validation and also a small sample set (10\%) for testing the instances randomly selected - appeared in Table 2 - to produce satisfactory accuracy. However, the prediction accuracy drastically reduces if the testing sample size and validation sample size is increased to $20 \%$ respectivelyappeared in Table 3 - and above, which reduces training sample size to $60 \%$.

Table 2. Effect of neuron count in hidden layer on accuracy of two-layer ANN.

\begin{tabular}{|c|c|c|c|}
\hline Num. & $\begin{array}{c}\text { Neuron } \\
\text { count( L1) }\end{array}$ & $\begin{array}{c}\text { Neuron } \\
\text { count (L2) }\end{array}$ & $\begin{array}{c}\text { Classification } \\
\text { accuracy (\%) }\end{array}$ \\
\hline 1 & 5 & 5 & 81.03 \\
\hline 2 & 10 & 5 & 91.83 \\
\hline 3 & 15 & 5 & 91.83 \\
\hline 4 & 20 & 5 & 92.85 \\
\hline 5 & 25 & 5 & 82.65 \\
\hline 6 & 30 & 5 & 82.65 \\
\hline 7 & 35 & 5 & 69.34 \\
\hline 8 & 40 & 5 & 50.27 \\
\hline 9 & 45 & 5 & 26.53 \\
\hline
\end{tabular}

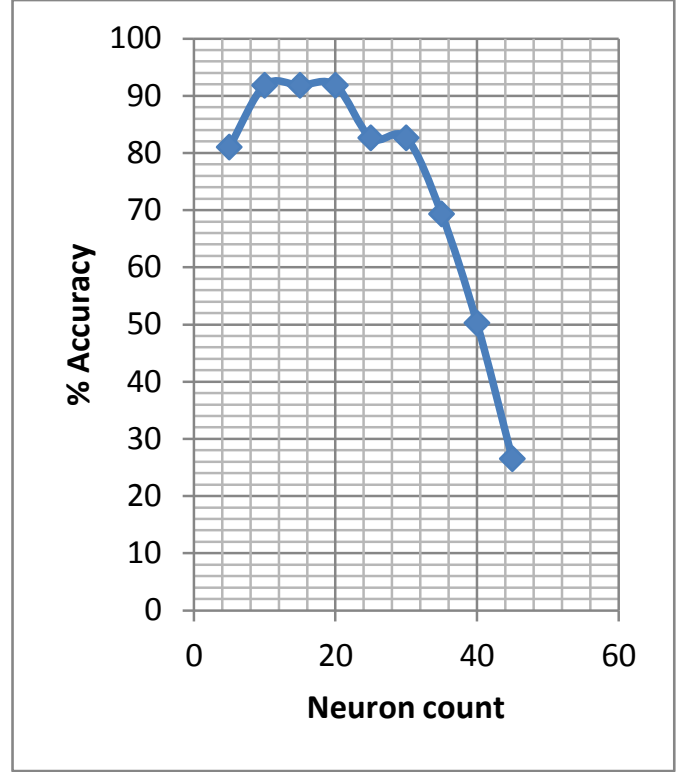

Fig 3: Effect of neuron count in hidden layers on classification accuracy of ANN

Table 3. Effect of multiple hidden layers added to ANN keeping count of neuron in additional hidden layers to five

\begin{tabular}{|c|c|c|}
\hline Num. & $\begin{array}{c}\text { Hidden layer } \\
\text { count }\end{array}$ & $\begin{array}{c}\text { Classification accuracy } \\
(\%)\end{array}$ \\
\hline 1 & 1 & 91.83 \\
\hline 2 & 2 & 92.85 \\
\hline 3 & 3 & 85.23 \\
\hline 4 & 4 & 72.44 \\
\hline 5 & 5 & 63.12 \\
\hline
\end{tabular}

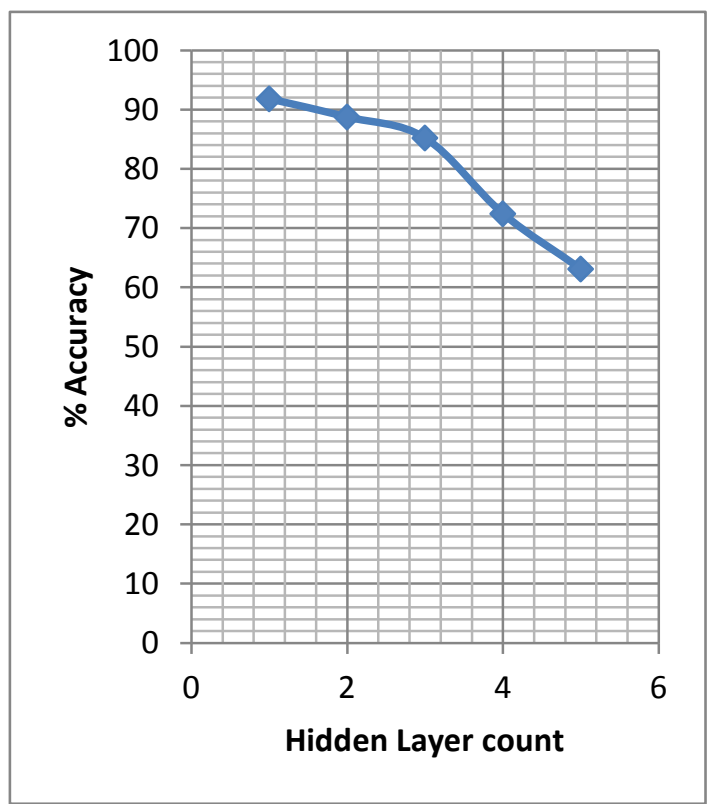

Fig 4: Effect of additional hidden layers on classification accuracy of ANN 
Table 4. Lists the effect of variation in test and validate sample size on prediction accuracy of two hidden layer ANN

\begin{tabular}{|c|c|c|c|}
\hline \multirow{2}{*}{ Validation } & \multicolumn{3}{|c|}{ Classification Accuracy of ANN (\%) } \\
\cline { 2 - 4 } Sample set (\%) & $\mathbf{0 . 1}$ & $\mathbf{0 . 2}$ & $\mathbf{0 . 3}$ \\
\hline 0.1 & 92.85 & 89.28 & 84.01 \\
\hline 0.2 & 91.83 & 89.28 & 84.13 \\
\hline 0.3 & 91.83 & 89.28 & 18.36 \\
\hline 0.4 & 11.22 & 15.81 & 10.24 \\
\hline
\end{tabular}

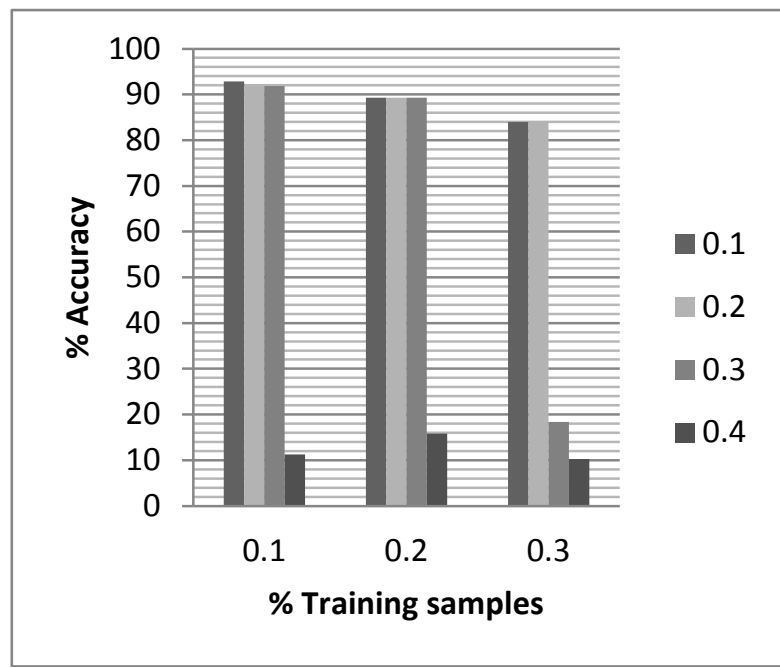

Fig 5. Effect of testing sample size \& validation sample size of classification of an ANN

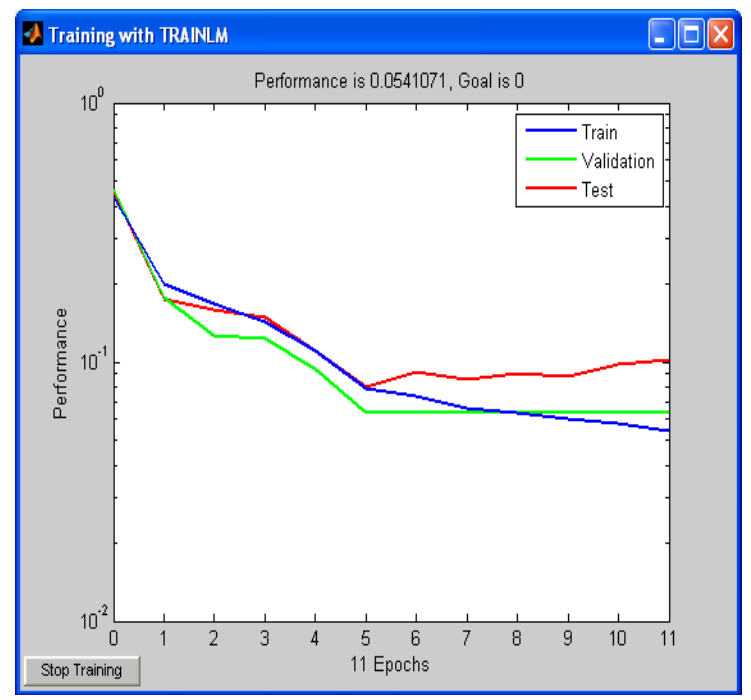

Fig 6: Network performance curves for the best feed forward ANN formed with two hidden layers

\section{CONCLUSIONS}

In this study, we explored the development of a decision support system (DSS) based on the artificial neural network
(ANN) architecture for medical diagnosis. The final network model appears to be reachable empirically performs acceptably as opined by practicing university doctors who would interpret the same symptoms (input) data to diagnose patients with speculated hypertension. The ANN system was trained by employing Levenberg-Marquardt back propagation algorithm, a well-established procedure in ANN technology. The number of hidden layers and the count of neurons in the hidden layers of a neural network does appear to play a critical role for correctly classifying the relevant pathology. Indeed, by suitable parameter selection done empirically as shown here may reduce the training time and deliver good accuracy for medical applications. Therefore, the ANN-based DSS appears to be performing at a level comparable to human experts for hypertension detection when the correct architecture, training and testing strategies are empirically established.

\section{REFERENCES}

[1] R Dybowski and v. Gant, (2007) Clinical Applications of Artificial Neural Networks, Cambridge University Press

[2] Bishop, C.M. (1995) Neural Networks for Pattern Recognition, Oxford: Oxford University Press. ISBN 019-853849-9

[3] X. Zhu, (2005),Semi-supervised learning literature survey, Dept. Computer. Sci.,Univ. Wisconsin-Madison, Madison, Tech. Rep. 1530.

[4] Rudiger W. Brause,(2000) Medical Analysis and Diagnosis by Neural Networks , URL: http://citeseerx.ist.psu.edu/viewdoc/download?doi=10.1. 1.1.2901\&rep=rep1\&type $=$ pdf.

[5] Lanzarini, L., \& Giusti, D. A. (2000). Pattern recognition in medical images using neural networks. IEEE Transaction on Image and Signal Processing Analysis

[6] Carretero, O. A., \& Oparil, S. (2000). Essential hypertension. Part I: Definition and etiology. Circulation, 101, 329-335.

[7] D. Gil, M. Johnsson, J. M. Garicia Chemizo, A.S. Paya and D.R. Fernandez,(2009) Application of Artificial Neural Networks in the diagnosis of Urological Dysfunctions, Expert systems with applications, Vol.36, No.3,2009, pp 5754-5760

[8] Mevlut Ture, Imran Kurt, A. Turhan Kurum, Kazim Ozdamar, (2005),Comparing classification techniques for predicting essential hypertension, , Expert Systems with Applications Vol. 29, pp 583-588

[9] Kuang-Hung Hsu, Chaochang Chiu, Nan-Hsing Chiu, Po-Chi Lee, Wen-Ko Chiu, Thu-Hua Liu, Chorng-Jer Hwang, (2011) A case-based classifier for hypertension detection, Knowledge-Based Systems 24 (2011) 33-39

[10] G. Zhang, P. Yan, H. Zhao and X.Zhang, (2008) A computer aided Diagnosis System in Mammography Using Artificial Neural networks, International conference on Biomedical Engineering and Informatics, Vol.2, pp.823-826

[11] Siddharth Jonathan, Shruthi Narayan, A Two Tier Neural inter-network based approach to Medical Diagnosis using k-Nearest Neighbor Classification for Diagnosis pruning

URL: http://infolab.stanford.edu/ jonsid/nimd.pdf 
[12] H. Kordylewski., D. Graupe, Kai Liu, (2001), A novel large-memory neural network as an aid in medical diagnosis applications, IEEE Transactions on Information Technology in Biomedicine, Sept. $2001 \mathrm{~V}$ $5 \# 3$.

[13] Hong Zhang, F. C. Lin, (1999), Medical diagnosis by the virtual physician, Proceedings of the $12^{\text {th }}$ IEEE Symposium on Computer-Based Medical Systems, June 99, pp. $296-302$.

[14] Danna Voth,(2005), Using AI to detect breast cancer, IEEE Intelligent Systems, Jan./Feb. 2005 vol. 20, no. 1, pp. 5-7
[15] Qeethara Kadhim Al-Shayea, Artificial Neural Networks in Medical Diagnosis, IJCSI International Journal of Computer Science Issues, 2011, (2):150-154

[16] Rahul Samant, Srikantha Rao . " Effects of Missing Data Imputation on Classifier Accuracy ", Vol.2 - Issue 11 (November - 2013), International Journal of Engineering Research \& Technology (IJERT), ISSN: 2278-0181 , pp 264-266

[17] Rahul Samant, Srikantha Rao . " A study on Feature Selection Methods in Medical Decision Support Systems ", Vol.2 - Issue 11 (November - 2013), International Journal of Engineering Research \& Technology (IJERT) ISSN: 2278-0181 , pp 615-619, 